Folge dieser Kreisreform verlor Zittau seine Funktion als Kreissitz. Heute gehört die Große Kreisstadt Löbau zum Landkreis Görlitz, nachdem in der Kreisreform vom 1. August 2008 die Landkreise Löbau-Zittau, der Niederschlesische Oberlausitzkreis und die Stadt Görlitz vereinigt wurden.

Mit der Verlagerung des Kreissitzes nach Zittau verlor Löbau einen Großteil seiner Zentralität und damit auch einen Teil seiner Wirtschaftskraft.

Löbau war als Sechsstädte-Stadt in das überregionale Fernstraßennetz der Via Regia eingebunden und damit mit den anderen Städten, Kamenz, Bautzen, Görlitz, Zittau und Lauban, verbunden. Zu Beginn des 19. Jahrhunderts wurde Löbau über Chausseen mit Bautzen, Görlitz und Zittau verbunden. Die alten Chausseen finden sich in der Bundesstraße B 6 Bautzen-Löbau-Görlitz und in der B 178 Löbau-Zittau wieder. Die vierspurige Bundesstraße 178n ist ein Rudiment zwischen der Autobahnanschlussstelle Weißenberg und Zittau. Vom Ende des Neubaus südlich von Särka bis zur Autobahn fehlen 6 ausgebaute Kilometer.

Zur Autobahn A 4 (Dresden-Görlitz-Breslau) sind es zur Anschlussstelle Weißenberg $16 \mathrm{~km}$, bis Görlitz über die B 6 im Osten $28 \mathrm{~km}$ oder über die B 6, am ertragreichen Blitzer von Plotzen vorbei, nach Bautzen $22 \mathrm{~km}$.

Löbau als ehemaliger Eisenbahnknoten liegt an der zweigleisigen Eisenbahnstrecke DresdenGörlitz, die zwischen 1843 und 1847 erbaut wurde und bis heute in Betrieb ist. Von Löbau gab es Verbindungen nach Ebersbach (1973), Zittau (1845-1848), Großpostwitz (1889-1928) und Radibor (1894-1906). Die Eisenbahntradi-

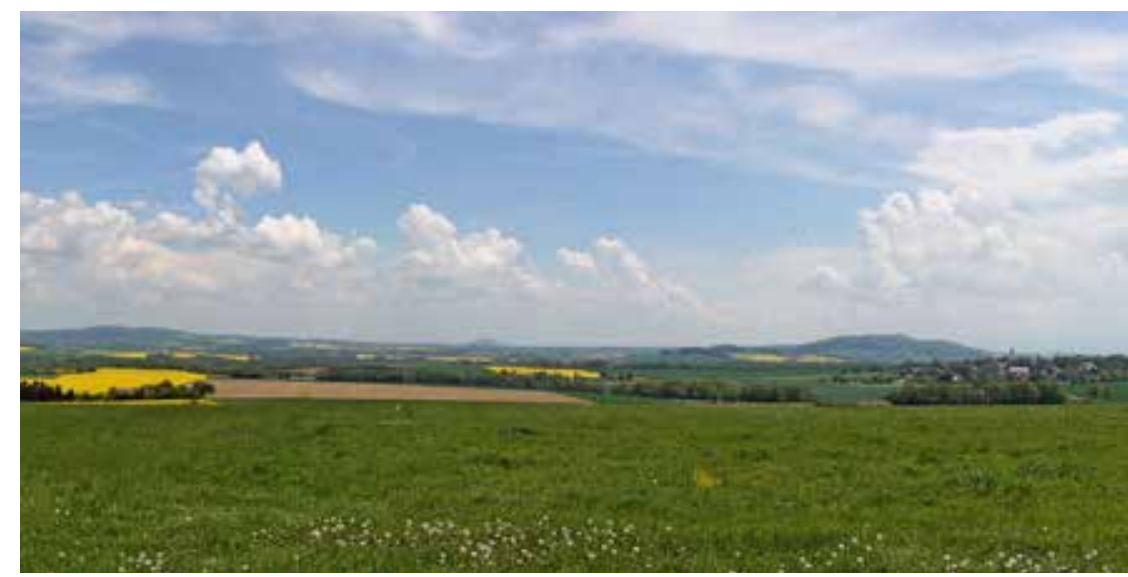

tion in Löbau wird durch die Ostsächsischen Eisenbahnfreunde mit dem Eisenbahnmuseum im Maschinenhaus Löbau aufrecht erhalten.

Der öffentliche Personennahverkehr in Löbau wird durch den Zweckverband Verkehrsverbund Oberlausitz-Niederschlesien (ZVON) geregelt. Über seine Mitgliedsunternehmen wird auch der Busverkehr in und um Löbau abgewickelt. Die Stadt Löbau hat 39 Bushaltestellen und wird in seinen Stadtteilen von sieben Linien bedient.

Seit April 2017 ist Löbau in dem internationalen Busnetz der Fa. Flixbus mit Linien nach Berlin, Prag, München und Breslau eingebunden. Der nächstliegende internationale Flughafen ist Dresden (über die A 4, 90 km/60 min.).

\section{Der Naturraum}

Das Stadtgebiet von Löbau gehört naturräumlich zur Makrochore des Oberlausitzer Gefildes $^{1}$, einem Teil des sächsischen Lößgefilde-
Blick von Wohla über die Löbauer Bucht zum Rotstein Foto: Uwe Ulrich Jäschke
1 Bastian, Olaf und Ralf-UweSyrbe: Naturräume in Sachsen - eine Übersicht. In Landesverein sächsischer schaftsgliederungen in Sachsen. Dresden, o.J., S. 19. Heimatschutz (Hrsg.): Land-

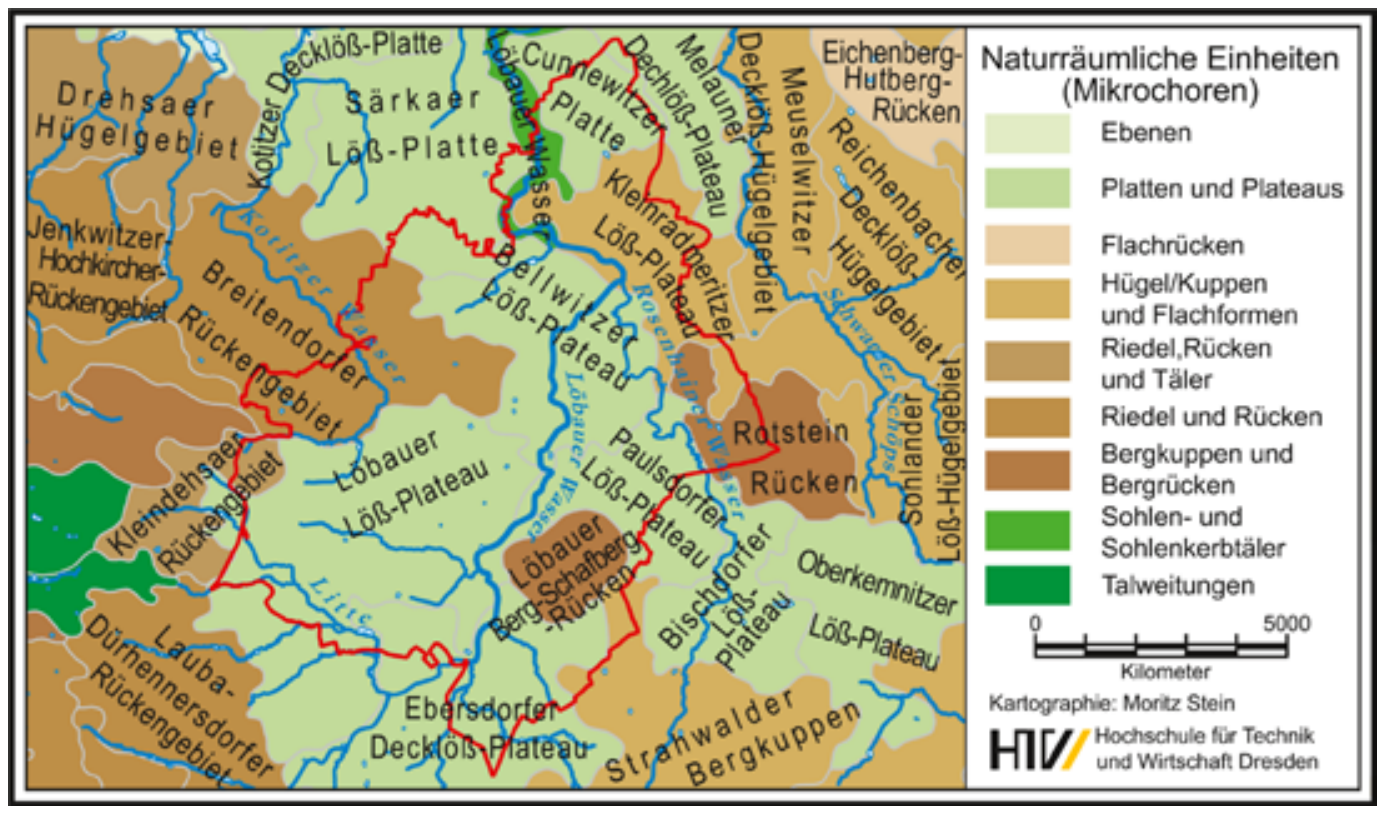

Naturräumliche Gliederung (Mikrochoren) des Löbauer Stadtgebietes.

Kartographie: Moritz Stein, 2016. 







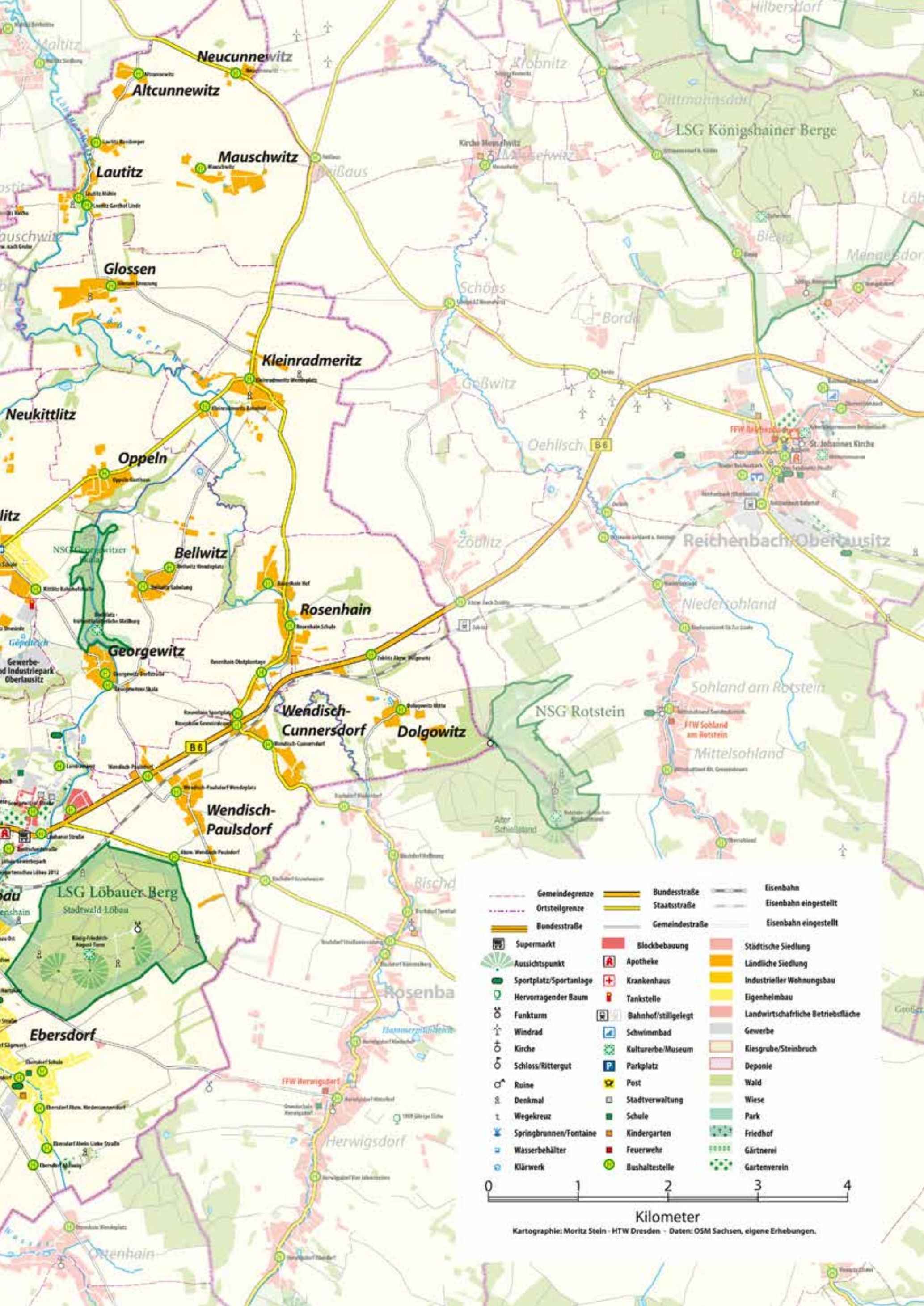




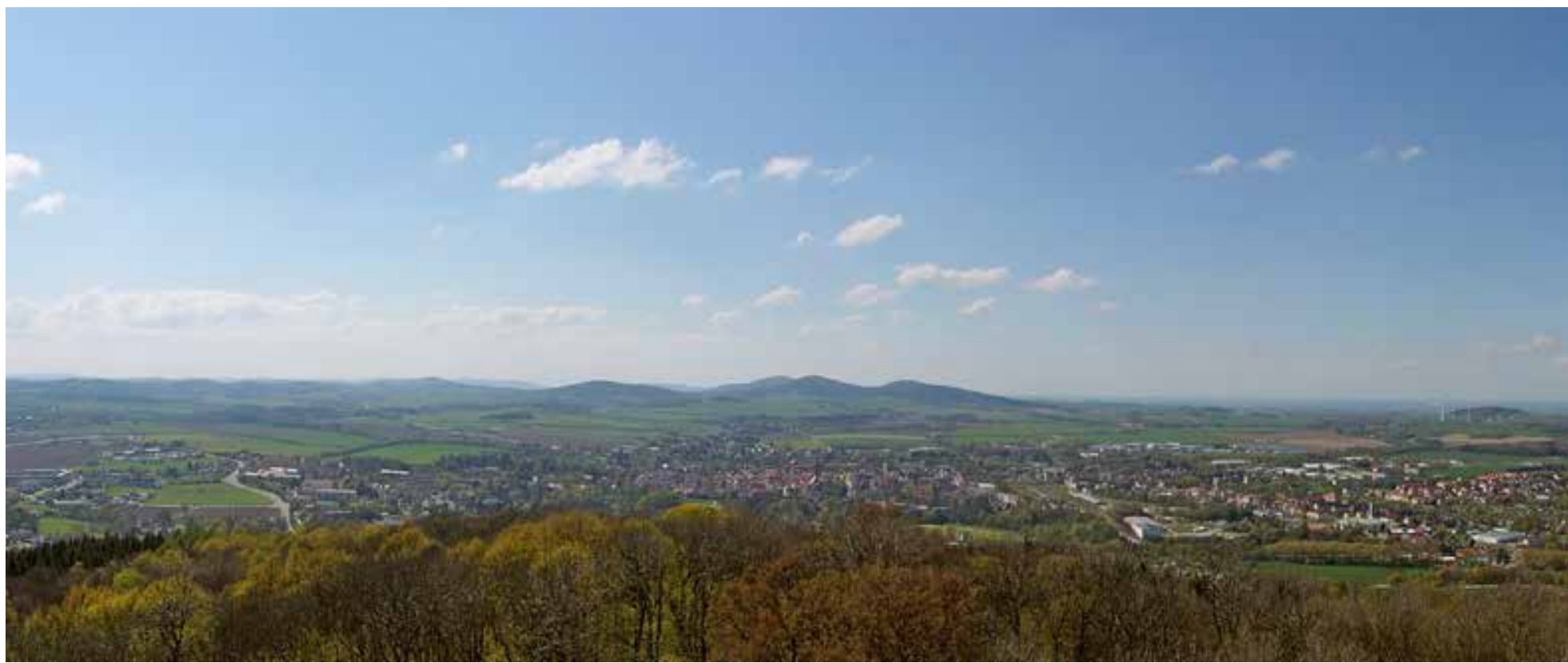

Oben: Blick vom König-FriedrichAugust-Turm (Löbauer Berg) nach Norden auf die Löbauer Bucht

Rechts: König-FriedrichAugust-Turm (Löbauer Berg) Fotos: Uwe Ulrich Jäschke, 2017

Klimadiagramm Löbau Daten: http://de.climatedata.org/location/8660/ Kartographie: Jäschke (2017): http://www2.htw-dresden.de/ jaeschke/Klimadiagramme/ Klima.html oder Hügellandes. Als östlichster Ausläufer umfasst es Löbau und die nähere Umgebung. Das Oberlausitzer Gefilde ist ein 12 bis $15 \mathrm{~km}$ breiter Streifen mit Lößbedeckung. Die Grundlage bildet der 540 bis 530 Millionen Jahre alte Granodiorit, der von einzelnen Vulkanereignissen durchschlagen wurde. Heute bilden die Granodioritflächen und eiszeitlichen Schmelzwasserbildungen der Saale- und Elstereiszeiten lössbedeckte Hügelgebiete und teilweise stark zerschnittene Plateaus in Höhenlagen zwischen 150 und $380 \mathrm{~m}$. Die Vulkankomplexe bilden steile und dicht bewaldete Berge. Um Löbau sind das im Süden die Quellkuppe des Löbauer Berges mit dem Schafbergrücken sowie im Osten der Rotsteinrücken, die der Mesochore Östliche Oberlausitz zuzuordnen sind. Nordwestlich beider Berge befindet sich die Löbauer Bucht, gebildet aus dem Ebersdorfer DecklößPlateau, dem Löbauer Löß-Plateau, dem Pellwitzer Löß-Plateau sowie dem Paulsdorfer LößPlateau. Die Löbauer Bucht wird von Hügeln und Rücken eingerahmt.

Die Mächtigkeit der entkalkten Lösse überschreitet selten 2 Meter. Die Jahresmitteltempe-

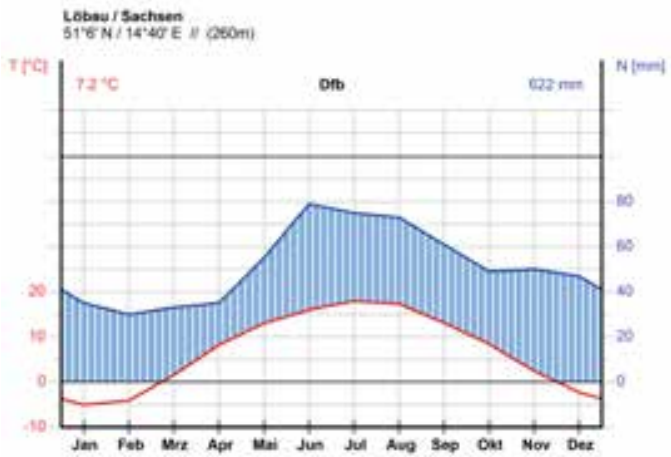

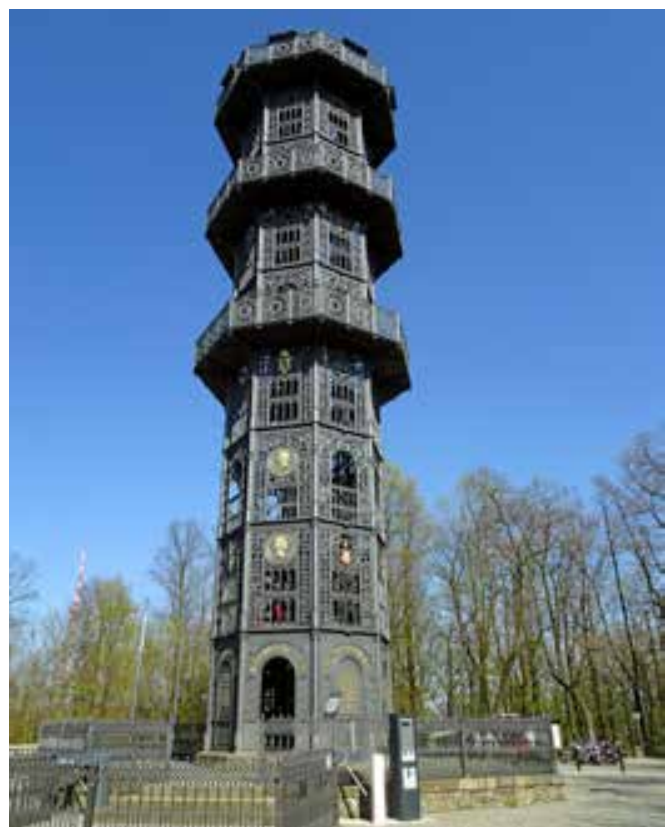

ratur liegt in Löbau bei $7,2^{\circ} \mathrm{C}$, der Jahresniederschlag bei $622 \mathrm{~mm}$.

\section{Schutzgebiete}

Der westliche Teil von Löbau im Bereich von Eiserode und Großdehsa wird vom Landschaftsschutzgebiet (LSG) Oberlausitzer Bergland berührt. Im Löbauer Stadtgebiet selbst liegt das LSG Löbauer Berg, das auch zugleich FFH-Gebiet Basalt- und Phonolithkuppen der östlichen Oberlausitz, Teilflächenbezeichnung: Löbauer Berg/ Schafberg ist. Weitere FFH-Gebiete im Stadtgebiet sind die Täler um Weißenberg, hier das Löbauer Wasser und Nebenbäche sowie das Buttermilchwasser. 


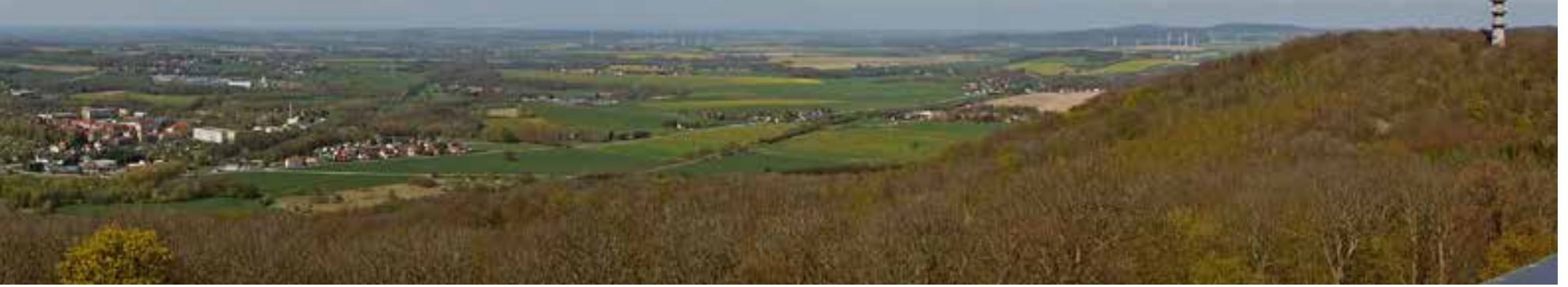

Teile der Gemarkung Kittlitz sind als Vogelschutzgebiet Feldgebiete in der östlichen Oberlausitz ausgewiesen. Naturschutzgebiete sind die Georgewitzer Skala, ein Felsental des Löbauer Wassers sowie knapp außerhalb der Stadtfläche mit Zugang über Dolgowitz der Rotstein mit neuem Aussichtsturm. Unter Denkmalschutz steht das Ensemble rund um den $21 \mathrm{~m}$ hohen gusseisernen Aussichtsturm auf dem Löbauer Berg.

\section{Eingemeindungen}

Die Stadt Löbau in ihrem Weichbild ist bis zum Ende des 19. Jahrhunderts territorial weitgehend konstant geblieben. Lediglich während des Oberlausitzer Pönfalles 1547 verlor die Stadt zeitweilig alle ihre für die städtische Versorgung wichtigen Landgüter. Doch diese konnten innerhalb kürzester Zeit zurückgewonnen werden. Am 31. Oktober 1549 wurden nach dem Pönfall eingezogene Güter durch Maximilian II. zurückgegeben. 1552 kauft Löbau die Gemeinde Oelsa zurück. $^{2}$

$\mathrm{Zu}$ Beginn des 19. Jahrhunderts war die Stadt Löbau Grundherr der Gemarkungen von Altlöbau, Ebersdorf, Oelsa und Tiefendorf.

Mit dem Gesetz über Ablösungen und Gemeinheitsteilungen und das Gesetz über die Landrentenbank vom 17. März 1832 konnten sich die Bauern aus der Gundabhängigkeit befreien und die Gemarkungen zu selbständigen Gemeinden entwickeln.

Die Entwicklung Löbaus von der Fernhandelsstadt an der Via Regia zur Industriestadt begann im 18. Jahrhundert mit der Leineweberei und entwickelte sich mit der Verkehrsanbindung über Chausseen an die umliegenden Zentren
Bautzen, Görlitz und Zittau sowie mit der Einbindung in das sächsische Eisenbahnnetz weiter. Mit der Einrichtung der Amtshauptmannschaft Löbau 1835 kamen weitere zentrale Funktionen nach Löbau, unter anderem Finanzdienstleistungen, Ausbildungsstätten, eine Garnison und andere öffentliche Einrichtungen.

In dieser Zeit des wirtschaftlichen Aufschwungs wurden erstmals unmittelbar an der Stadt gelegene Gemeinden eingemeindet. Als erstes 1844 Tiefendorf, ein Gassendorf am heutigen Stadtrand um die Herwigsdorfer Straße. Nach dem Stadtbrand von 1710 war hier ein Ziegelei errichtet worden, später ein Luftbad zur Naherholung. Heute ist es ein Wohngebiet mit Gewerbe.

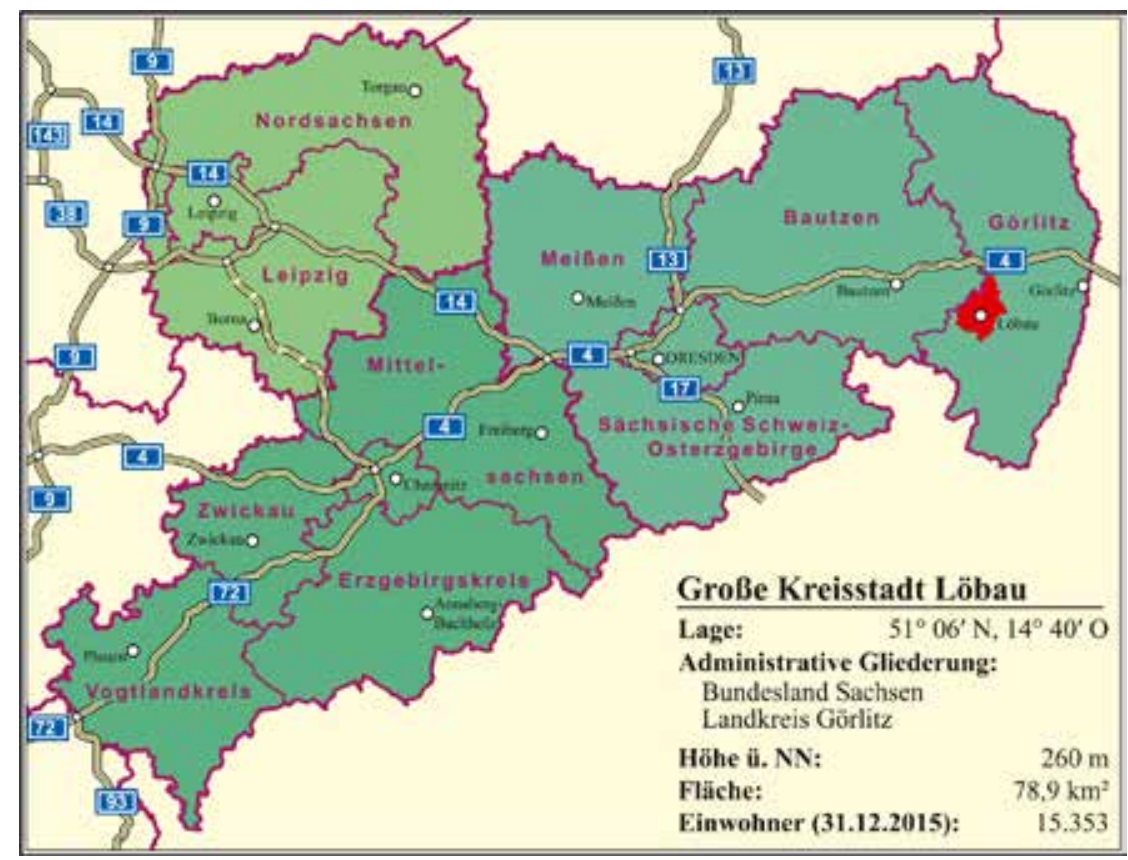

2 Stadtverwaltung Löbau (Hrsg.): Chronik der Stadt Löbau. Löbau 2001, S. 93 ff.

Löbau: Administrative Einordnung Kartographie: Uwe Ulrich Jäschke, 2017 
Viadukt mit Stadtkirche Altlöbau

Vierseithof in Oelsa

Weiler Peschen

Nechen
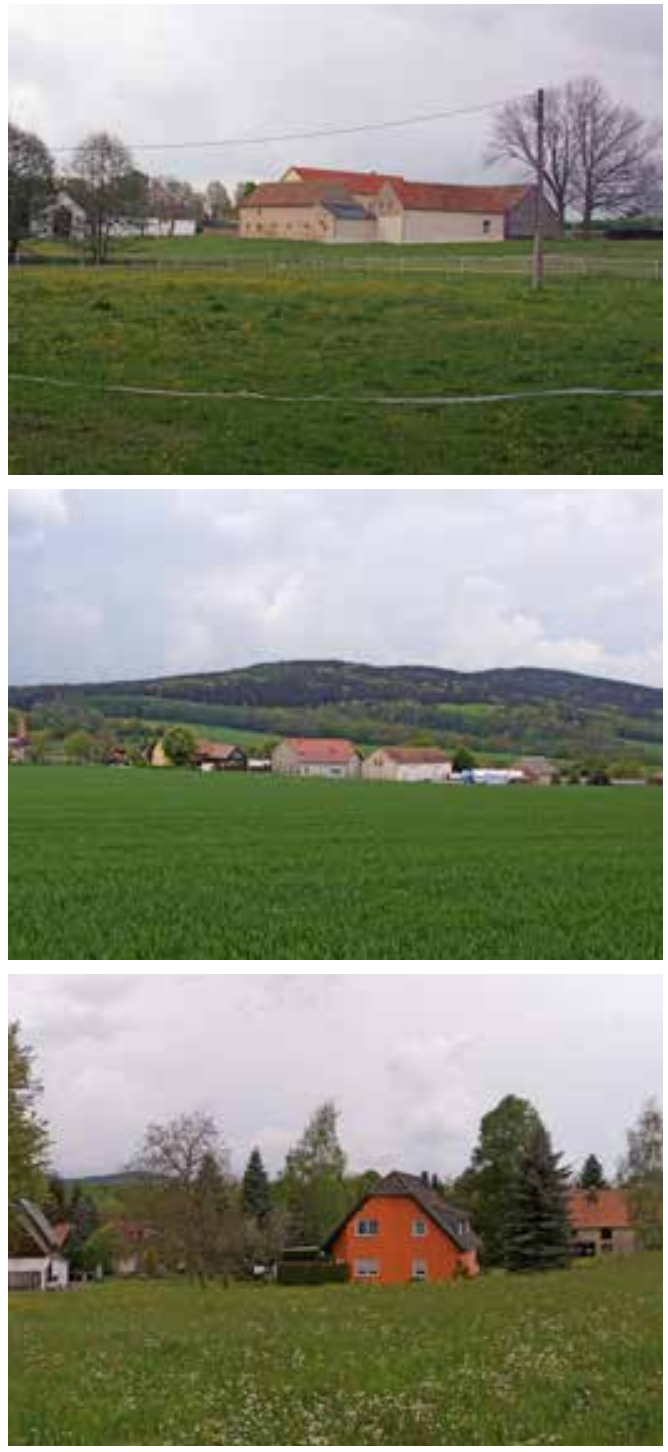

Großdehsa Fotos: Uwe Ulrich Jäschke, 2017

3 Stadtverwaltung Löbau (Hrsg.): Chronik der Stadt Löbau. Löbau 2001, S. 93 ff.

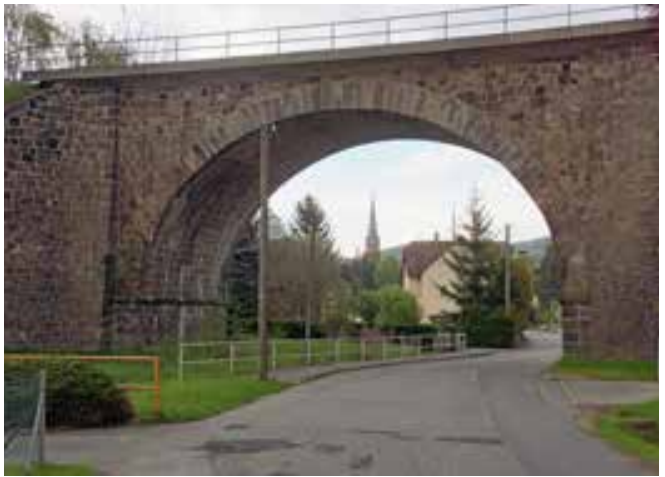

Die zweite Eingemeindung erfolgte 1888 mit Körbigsdorf, einem Gutsweiler mit Rittergut am östlichen Ortsausgang an der B 6 gelegen. Beide ehemaligen Gemeinden sind heute im Stadtbild nicht mehr erkennbar.

Als nächstes wurde 1934 in unmittelbarer Stadtnähe Altlöbau (Stary Lubij) eingemeindet. Das Waldhufendorf im Tal Seltenrein war ab 1421 teilweise, ab 1438 vollständig bis zur Ablösung der Grundherrschaft 1838 ein stadtmitleidendes Ratsdorf. Bis heute hat es trotz der Stadtnähe mit seinen an den Talhängen liegenden Bauernhöfen und Umgebindehäusern seinen dörflichen Charakter erhalten.

Wie Altlöbau liegt auch Oelsa (Wolešnica) im Seltenreintal westlich von Löbau. Im Laufe des 15. Jahrhunderts kaufte der Löbauer Rat das Waldhufendorf auf, das ab 1838 wieder selbständige Gemeinde wurde. Der Quellbereich der Seltenrein versorgte die Stadt Löbau mit Röhrenwasser. 1979 wurde der Ort nach Löbau eingemeindet. Das Platzdorf Eiserode (Njeznarowy) ist wohl während der Deutschen Ostkolonisation entstanden. In einer Urkunde von 1354 wurde die Grundherrschaft auf das Kloster Marienstern übertragen. Das Ortsbild prägen große Dreiund Vierseithöfe, am südlichen Ortsrand befindet sich ein neuzeitlicher Ausbau mit 11 Einfamilienhäusern. 1994 wurde der Ort mit seinen Ortsteilen Peschen und Nechen nach Löbau eingemeindet.

Der Ortsteil Peschen (Stwěšin) mit seinen zwei großen Vierseithöfen wird im Historischen Ortsverzeichnis HOV als lockerer Rundweiler bezeichnet. Seit dem 15. Jahrhundert gehörte der Ort zu den Ratsdörfern der Stadt Bautzen und ist wohl 1838 Eiserode zugeschlagen worden. Der Freiraum zwischen Peschen und Eiserode wird seit 1971 durch den Komplex der Mastanlagen der früheren LPG Einheit Eiserode belegt.

Der Ortsteil Nechen (Njechań), 1306 der Löbauer Ortsgerichtsbarkeit unterstellt, wurde in der Folge ein Bautzener Ratsdorf. Die im $\mathrm{HOV}^{3}$ genannte Form des Rundweilers ist im Siedlungsbild nicht mehr zu erkennen, da die historische Bebauung in der zweiten Hälfte des 20. Jahrhunderts verloren ging. 1939 wurde der Ort nach Eiserode eingemeindet. Seit 1953 wurde dort ein Zuchtbetrieb für MerinoFleischschafe betrieben, dessen Herde seit 1969 als Elite-Stammherde geführt wurde.

Das Waldhufendorf Großdehsa (Dažin) liegt an der westlichen Stadtgrenze von Löbau und wurde 1242 erstmals erwähnt. Durch Kauf befand es sich im Besitz des Domstiftes Bautzen und zum Teil der Landvogtei Bautzen. Der Ort wurde 1994 nach Löbau eingemeindet und hat bis heute seinen bäuerlichen Charakter erhalten. 
1994 wurde die Landgemeinde Rosenhain (Róžany) mit den dazugehörigen Gemeindeteilen nach Löbau eingemeindet. Das Waldhufendorf wurde 1317 erstmals urkundlich erwähnt und war in der späteren Zeit trotz eigenem Rittergut (1619) verschiedenen Grundherrschaften zugeordnet. Neben dem Rittergut waren drei Mühlen am Rosenhainer Wasser von wirtschaftlicher Bedeutung. Am nördlichen Ortsausgang stehen Häuser eines modernen Ortsausbaus.

1939 wurden die Gemeinden Wendisch-Paulsdorf (Serbske Pawlecy) und Wendisch-Cunnersdorf (Serbske Kundraćicy) nach Rosenhain eingemeindet und in Rosenhain II und III umbenannt, nach 1945 in Rosenhain B und C.

Das HOV sieht in Wendisch-Paulsdorf eine platzartige Gutssiedlung mit Gutsblockflur, die seit 1317 dem Weichbild der Stadt Löbau zuzurechnen ist. Das Rittergut wird 1630 als Grundherrschaft erstmals genannt. Neben älteren

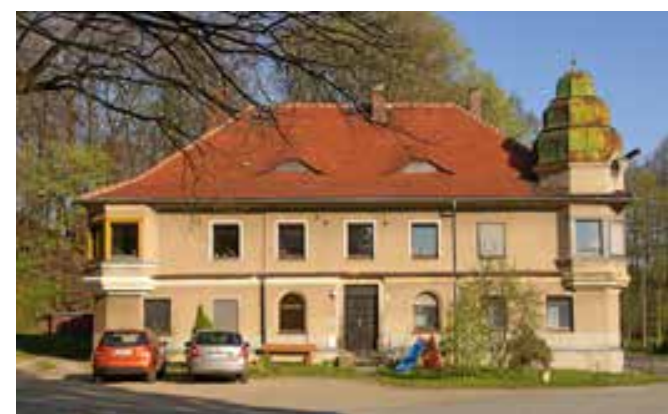

Rittergut Rosenhain

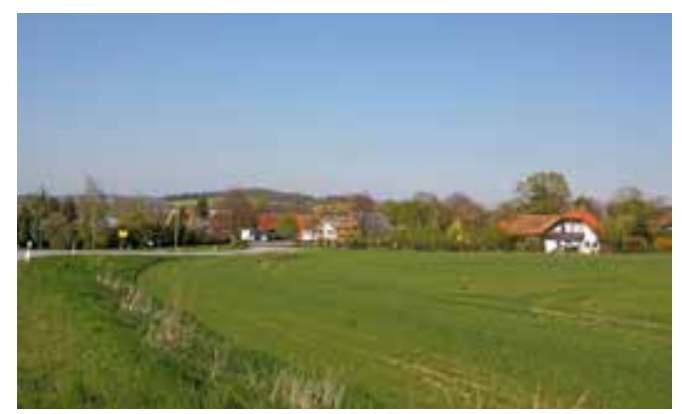

Ortseingang Wendisch-Paulsdorf

Fotos: Uwe Ulrich Jäschke, 2017

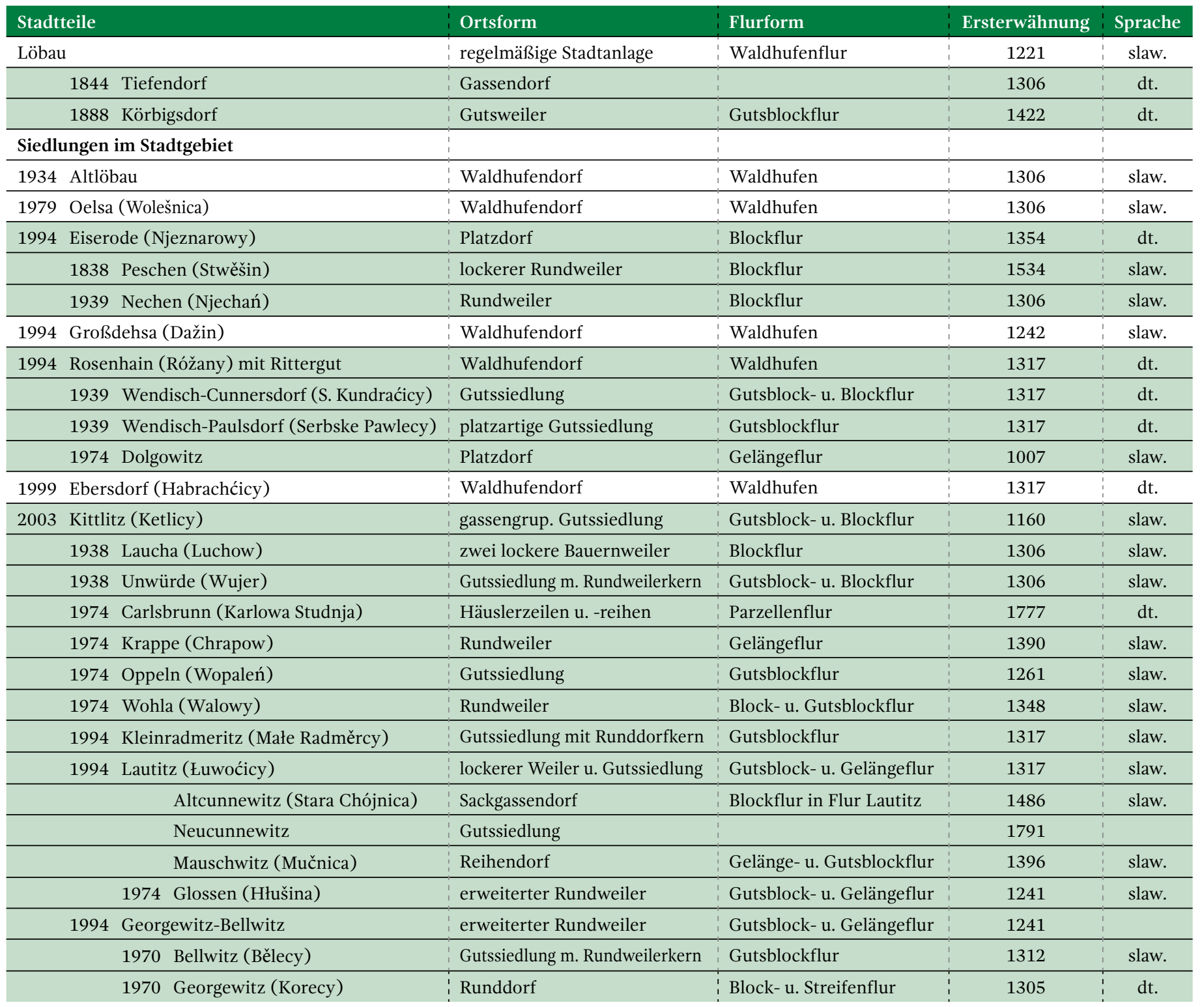




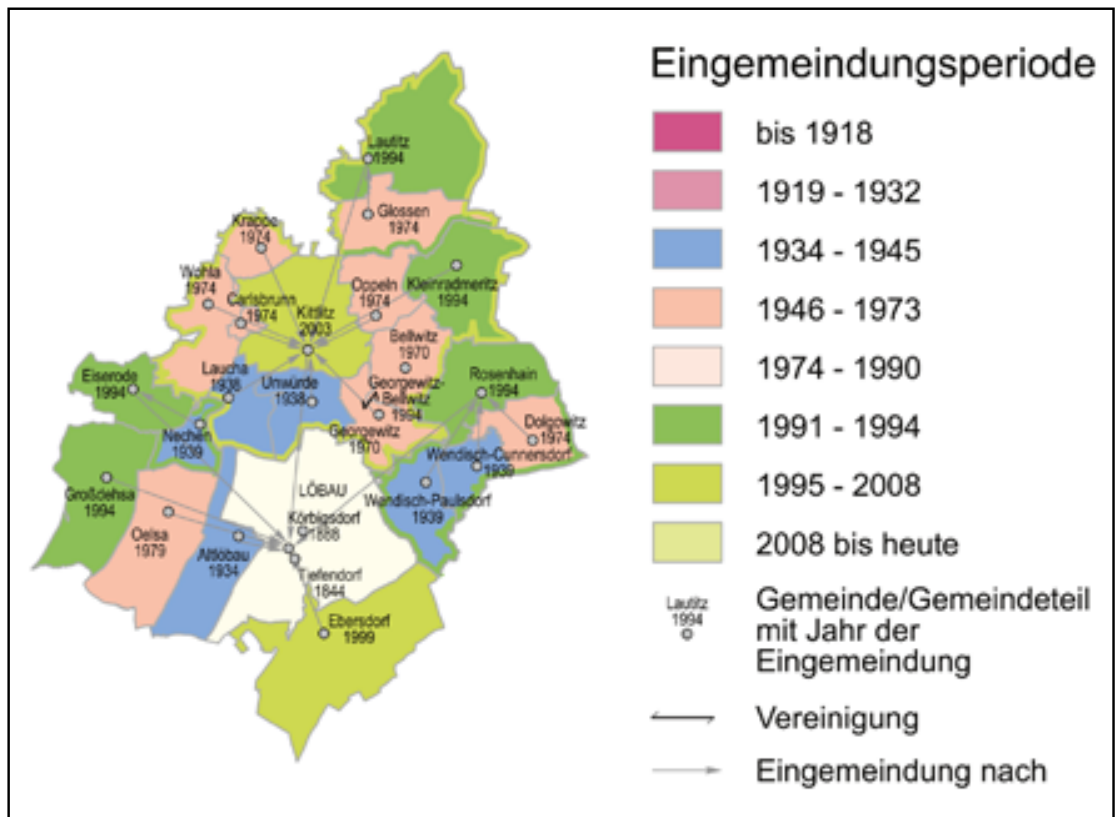

Löbau: Eingemeindungen Kartographie: Moritz Stein

Rittergut Wendisch-Cunnersdorf (links), Untere Dorfstraße in Ebersdorf (rechts)

Dreiseithof in Dolgowitz (links), Dorfkirche in Kittlitz (rechts) Fotos: Uwe Ulrich Jäschke, 2017 landwirtschaftlichen Gebäuden besteht der heutige Stadtteil von Löbau aus neuzeitlichen Einfamilienhäusern.

Wie die meisten Dörfer in der Oberlausitz gehörte Wendisch-Cunnersdorf landsässigen Adelsgeschlechtern, d. h. die Dorfflur war durch verschiedene Grundherrschaften zersplittert. Erst 1777 wurde im Ort ein eigenständiges Rittergut geschaffen.

Das Gemeindegebiet von Rosenhain wurde 1974 durch die Eingemeindung des Ortsteils Dolgowitz (Dołhaćicy) erweitert. Nach dem HOV ein
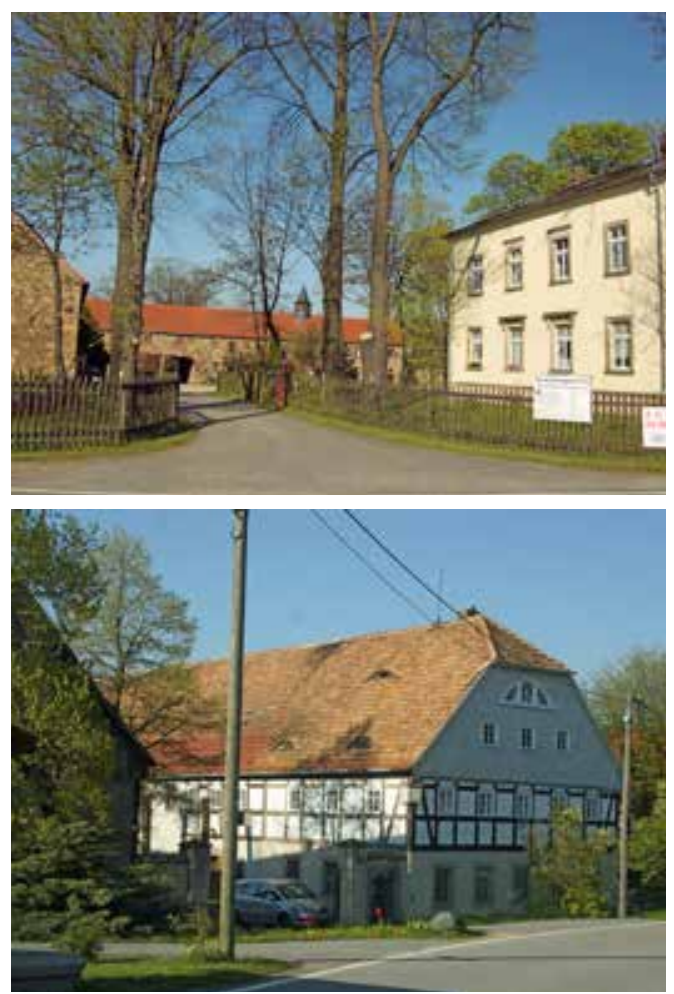

Platzdorf, wurde es 1241 erstmals erwähnt und gehörte zur Grundherrschaft Rittergut Unwürde. Im Ort soll kleinbäuerliche Landwirtschaft betrieben worden sein. Allerdings weisen schon die sächsischen Meilenblätter Blatt 420 von 1824 sechs große Drei- und Vierseithöfe an einer Durchgangsstraße auf, was auf ein Gassendorf mit großbäuerlichen Strukturen hinweist.

Bis heute haben die ehemaligen Gemeindeteile von Rosenhain in ihrer Bausubstanz eine bäuerlich Grundstruktur.

Das 1999 eingemeindete Ebersdorf (Habrachćicy) liegt im Süden des Löbauer Stadtgebietes. 1317 ersterwähnt war das Waldhufendorf bis 1531 im Besitz des landsässigen Adels, dann war es Ratsdorf der Stadt Löbau. Nach dem Pönfall konnte die Stadt Löbau den Ort erst 1576 zurückkaufen. Bekannt ist es auch durch die Schlacht bei Ebersdorf 1813.

Wirtschaftlich geprägt war das Waldhufendorf ab dem 17. Jahrhundert durch die sich entwickelnde Hausweberei, die sich im Ortsbild durch zahlreiche Umgebindehäuser dokumentiert. Am Nordende des Dorfes bildete sich im 18./19. Jahrhundert der Mühlenstandort Liebesdörfel. Durch die Stadtnähe Löbau entwickelten sich in der Gemarkung Wohnbereiche mit Ein- und Mehrfamilienhäusern.

Die Landgemeinde Kittlitz (Ketlicy) liegt nördlich von Löbau und war als eines der ältesten Orte der Oberlausitz ein Gegenpol zur Stadt. Als Missions- und Pfarrgemeinde betreute der Ort über 31 Gemeinden in der Region. Grund-
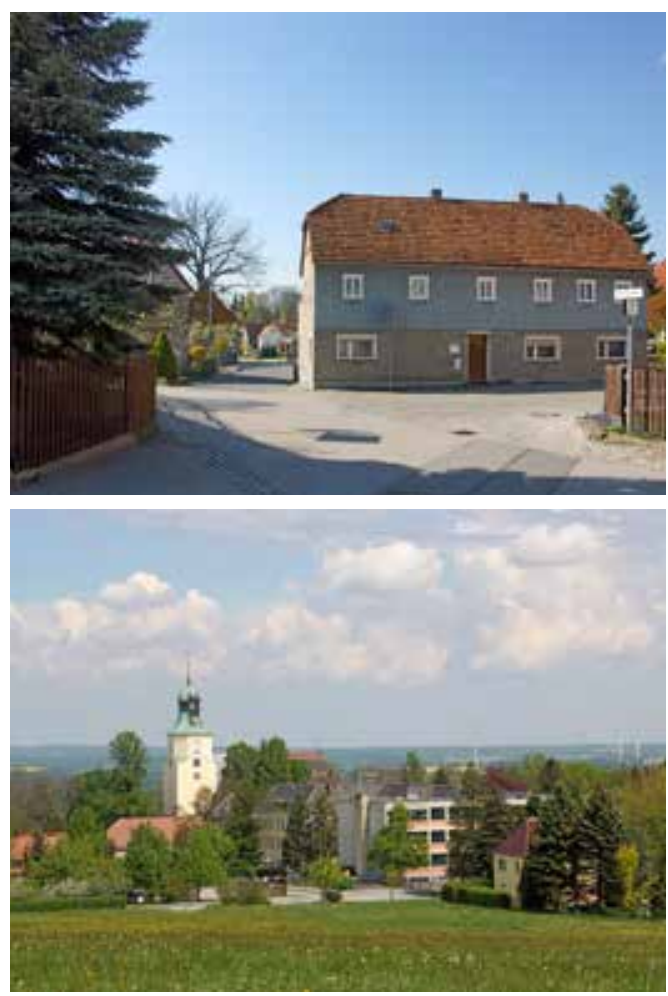

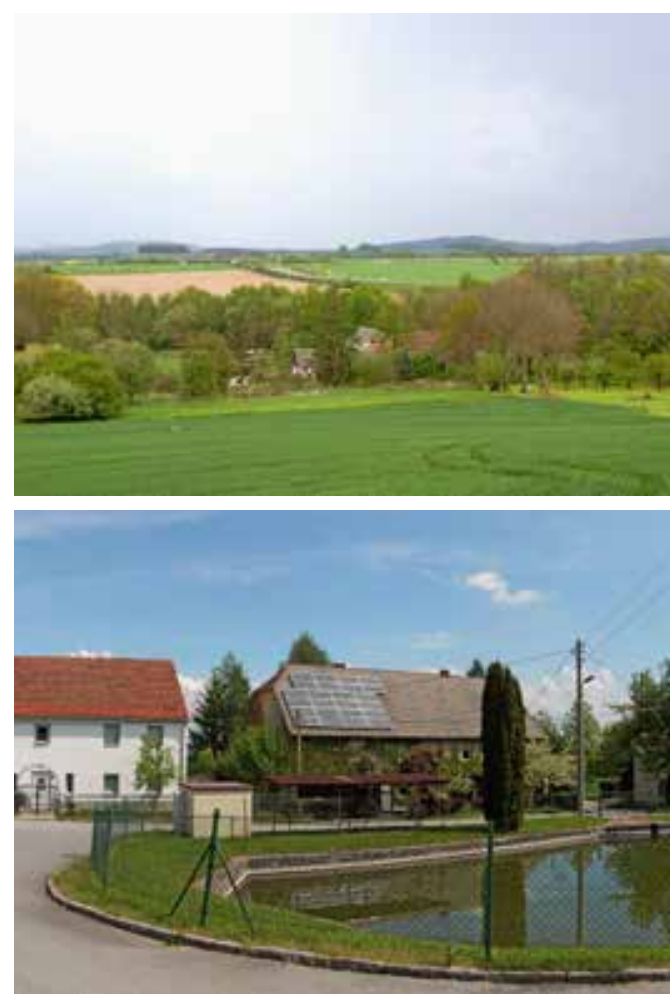

herr des landsässigen Adels war der jeweilige Besitzer des gleichnamigen Rittergutes.

Der Ortsteil Neukittlitz war eine Ausgründung im 18. Jahrhundert des Kittlitzer Rittergutes als Ziegelei. Eine Tagelöhnersiedlung mit 12 Wohnhäusern wurde angelegt. 1912 wurde die Ansiedlung durch ein Vorwerk erweitert.

Mit all seinen Eingemeindungen bestand Kittlitz 2003 bei der Eingemeindung aus 16 Ortsteilen auf rund $34 \mathrm{~km}^{2}$.

Die ersten Eingemeindungen nach Kittlitz waren 1938 die Nachbargemeinden Laucha und Unwürde.

Laucha (Luchow) besteht aus zwei Bauernweilern. Als Löbauer Weichbilddorf wurde es 1306 erstmals erwähnt. Ab 1491 wird als Grundherr ein Herr von Kittlitz genannt, der mit der Stadt Löbau in einem Rechtsstreit um den Ort lag.

Die Gutssiedlung Unwürde (Wujer) wurde 1308 erstmals genannt und wurde zu einem Rittergut ausgebaut. Die Flächen des Ritterguts sind nach 1945 an Neubauern verteilt worden. Heute ist der Ort mit Kittlitz verschmolzen 1974 wurden vier weitere Gemeinden nach Kittlitz eingemeindet.

Carlsbrunn (Karlowa Studnja) ist eine 1763 gegründete Handwerkersiedlung für evangelische Glaubensflüchtlinge aus Böhmen. Nach dem 2. Weltkrieg wandelte sich das Dorf zu einer Arbeiterwohnsiedlung.

Krappe (Chrapow), seit 1390 bekannt, gilt als als Weiler mit landwirtschaftlicher Prägung. Vor 1869 gehörte es zum Rittergut Nostitz.

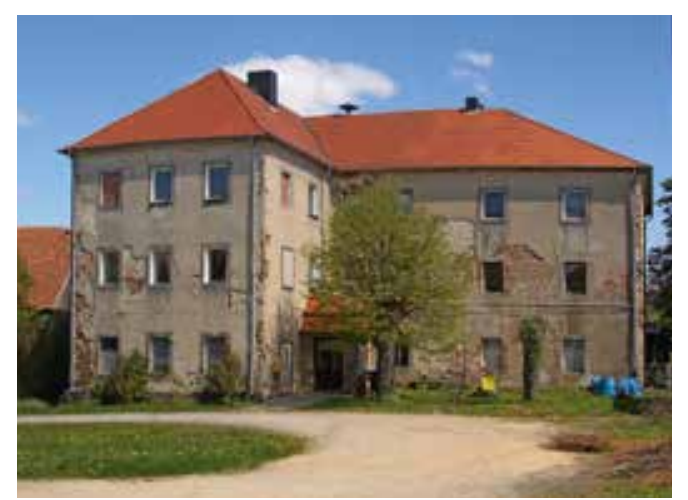

Weiler Laucha (links), Rittergut Oppeln (rechts)
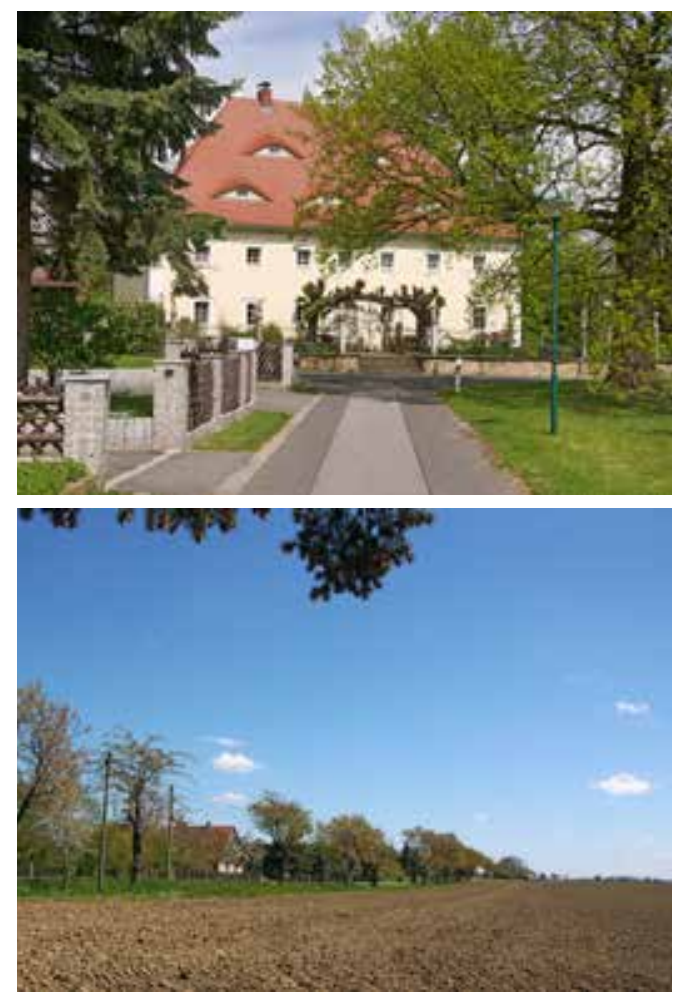

Kleinradmeritz, Neubauernsiedlung an der Zoblitzer Straße

Fotos: Uwe Ulrich Jäschke, 2017
Ortsmittelpunkt Carlsbrunn (links), Rittergut in Wohla, heute Hotel (rechts)
Die Gutssiedlung Oppeln (Wopaleń), 1298 ersterwähnt, war der Sitz zweier Rittergüter, eines in Oppeln, das andere im Ortsteil Kalkreuth. Das Oppelner Rittergut ist als Wohnhaus erhalten. Verkehrstechnisch lag Oppel an einem Abzweig der Via Regia Lusatiae Superioris mit einer Furt durch das Löbauer Wasser.

Wohla (Walowy), vom HOV als Rundweiler bezeichnet, liegt am Hang des Wohlaer Berges und hat einige einzelstehende Anwesen in seiner Peripherie. Die Form des Rundweilers lässt sich in der Dresdener Ausgabe der Sächsischen Meilenblätter nicht erkennen. Grundherren des Rittergutes und des Dorfes waren erst die Kirche in Kittlitz und später der landsässige Adel der Region.

Kleinradmeritz (Małe Radměrcy), 1261 erstmals erwähnt, ist eine Gutssiedlung mit den peripheren Einzelsiedlungen Buda und Fritzkau und wurde 1994 nach Kittlitz eingemeindet. Als Besonderheit ist hervorzuheben, dass ab 1849 
Mühle am Löbauer Wasser mit Hopfenanlage in Lautitz

Neucunnewitz

Schloss Glossen, ehemals Rehabilitationsklinik für Psychosomatik und Psychomotorik Fotos: Uwe Ulrich Jäschke, 2017 das Dorf nicht mehr zum landsässigen Adel, sondern bis 1913 zum Fideikommiss der Großherzoglichen Familie von Sachsen-Weimar-
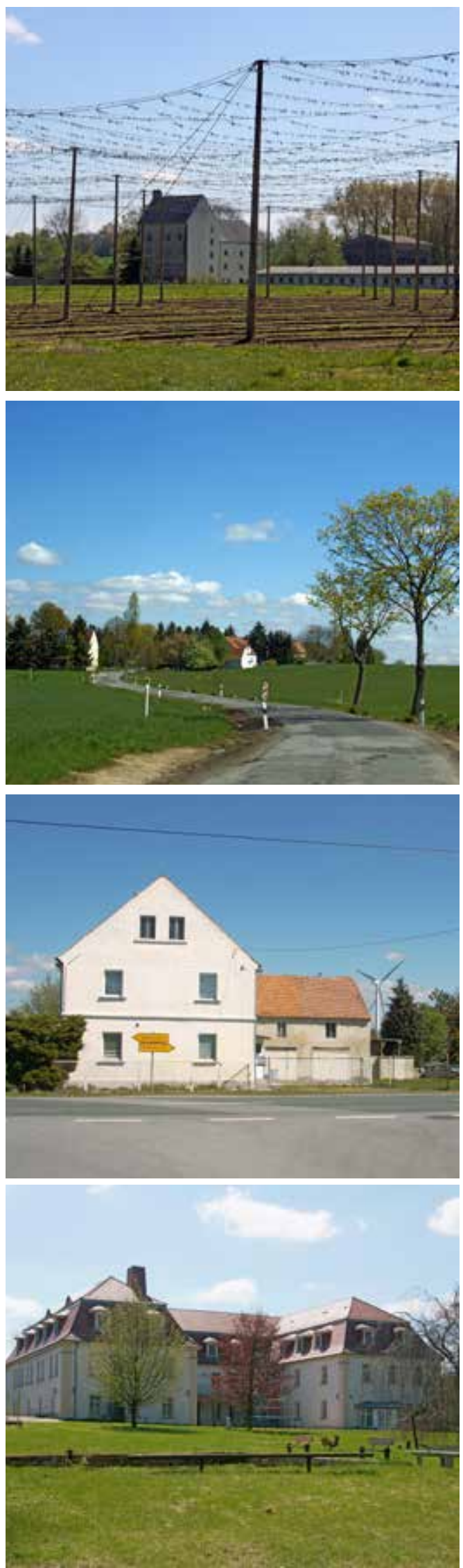

Eisenach gehörte. Nach der Enteignung des Rittergutes wurden die Flächen an ortsansässige Bauern und Umsiedler aus dem schlesischen Seichau verteilt. Für die Neubauern wurden im Norden des Dorfes Neusiedlerstellen geschaffen.

Lautitz (Łuwoćicy) mit seinen Ortsteilen Altund Neucunnewitz liegt im Norden des Löbauer Stadtgebiets. Es wird als Gutssiedlung mit lockerem Bauernweiler bezeichnet. Das Dorf wird in einer Gerichtsurkunde 1206 mit Henricus de Lutiz als Zeuge erwähnt. 1318 wird ein Herrensitz genannt. Im 19. Jahrhundert wurde in Lautitz der Zuckerrübenanbau in der Oberlausitz eingeführt. Nach dem Zweiten Weltkrieg wurde das Rittergut volkseigenes Gut mit Lehrlingsausbildung, später der LPG Löbau-Nord zugeschlagen. Ein Zweig der Landwirtschaft war und ist der Hopfenanbau. Der Ort hatte bis zu seiner Eingemeindung nach Kittlitz 1994 Versorgungseinrichtungen für den unmittelbaren Nahbereich.

Das Sackgassendorf Altcunnewitz (Stara Chójnica) liegt als kleines Bauerndorf in der Flur von Lautitz. Der Ortsteil Neucunnewitz, eine ehemalige Poststation, befindet sich östlich davon an der alten Poststraße nach Breslau.

Das Dorf Mauschwitz (Mučnica) wird vom HOV als Reihendorf bezeichnet und zieht sich in lockerer Bebauung an einem Seitenbach des Löbauer Wassers entlang.

Ende des 14. Jahrhunderts wurde das örtliche Rittergut in ein Vorwerk umgewandelt und aufgeteilt. Der westliche Teil gehörte zum Rittergut Lautitz im Bautzener Kreis, der östliche Teil wurde vom Rittergut Glossen im Görlitzer Kreis verwaltet. Nach 1945 wurde das Vorwerk enteignet und das Land an Neusiedler verteilt.

Mit der Eingemeindung von Glossen (Hłušina) 1974 nach Lautitz wurden die beiden Gemarkungen wieder vereint. Das Rittergut Glossen, nach einem Brand 1688 erbaut, prägt auch heute den erweiterten Rundweiler. Das Rittergut Glossen wurde 1946 enteignet und die Flächen verteilt. Das Schloss hatte verschiedenste Funktionen, von Notunterkünften über ein FDGBSchulungsheim, ein Kinderkurheim bis zur heutigen Nutzung als Rehabilitationsklinik für Psychosomatik und Psychomotorik. Der Dorfteil Hasenberg mit drei Häusern wurde 1831 vom Gutsbesitzer Schmaltz angelegt.

1970 wurde der Ort Georgewitz-Bellwitz durch die Vereinigung der Rundsiedlung Georgewitz (Korecy) und der Gutssiedlung Bellwitz (Bělecy) gebildet. Beide Orte gehörten wechselnden landsässigen Grundherrschaften Das Runddorf Georgewitz (Korecy) wurde 
1306 der Grundherrschaft der Stadt Löbau unterstellt und wechselte von da an mehrmals den Grundherrn bis es 1549 von Ulrich von Nostitz gekauft und dem Rittergut Unwürde zugeschlagen wurde. Auch später wechselte das Dorf häufig seinen Grundherrn. Heute sieht das Dorf trotz seiner bäuerlichen Grundstruktur ungepflegt aus.

Bellwitz (Bělecy) ist eine 1312 erstmals erwähnte Gutssiedlung, die 1572 zum Rittergut erhoben wurde. Wie alle Rittergüter wurde auch das Rittergut Bellwitz nach 1945 enteignet und das Land an 35 landlose und landarme Bauern vergeben. So entstanden im Dorf 14 Neubauernhöfe. Das Schloss steht heute, nach unterschiedlicher Nutzung im sanierungsbedürftigen Zustand leer.

Nördlich liegt der Dorfausbau Niederbellwitz mit vier Neubauernhöfen und Eigenheimen sowie einem LPG-Rinderstall.

\section{Bevölkerung}

Die Bevölkerungsentwicklung der Stadt Löbau und deren Stadtteile vor- und nach der Eingemeindung zeigt die nebenstehende Abbildung. $\mathrm{Zu}$ bemerken ist, dass ein fast linearer Anstieg der Wohnbevölkerung von 1834 (um 2.500 Einwohner) bis vor den Zweiten Weltkrieg (um 15.000 Einwohner) stattgefunden hat. Auch die wenigen Eingemeindungen haben auf Grund der geringen Einwohnerzahlen der ländlichen Orte im Umland die Bevölkerungskurve nicht beeinflusst. Der Anstieg von 1945 nach 1950 ist auf die Ansiedlung der zahlreichen Flüchtlinge und Vertriebenen aus den deutschen Ostgebieten, mehr im ländlichen Bereich als in der Stadt selbst, zurückzuführen.

Die Abbildung der Bevölkerungsentwicklung zeigt allerdings ebenso deutlich, dass die Abwanderung der Bevölkerung aus der Region Löbau nicht die Folge der politischen Wende von 1989 ist, sondern schon nach 1950 eingesetzt, sich aber nach 1989 verstärkt hat.

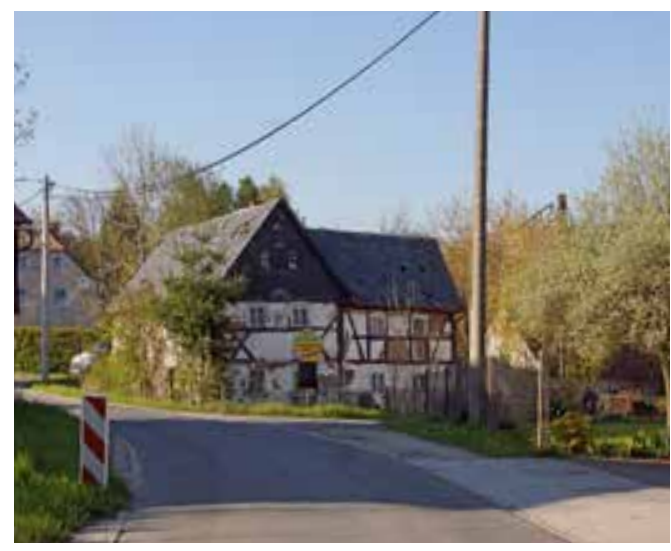

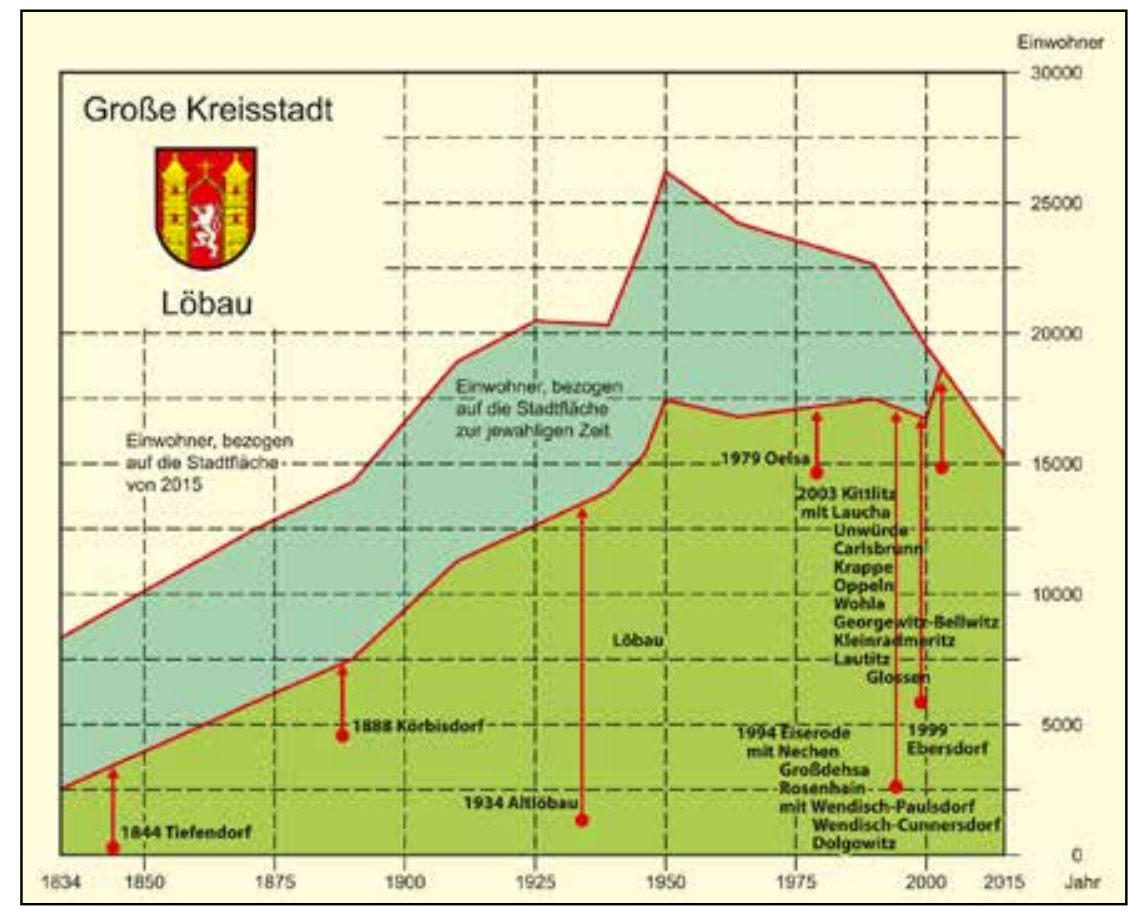

Dabei konnte die Stadt Löbau zum jeweiligen Gebietsstand auf einem ähnlichen Niveau stagnieren, ja sogar kurzfristig durch die Eingemeindung der stark verschuldeten Gemeinde Kittlitz mit deren 14 Gemeindeteilen steigern. Letztendlich setzt sich der starke Bevölkerungsverlust fort.

Der festgestellte Bevölkerungsverlust, der auch im starken Rückgang der sorbischen Sprache festzustellen ist, deutet auf eine schwindende Lebensqualität in Ostsachsen hin. Fehlende Industriearbeitsplätze, mangelnde Verkehrsanbindung, Verlust von wirtschaftlichem Hinterland jenseits der Neiße und damit geringe Einkommen führen zur Schwächung des Dienstleistungssektors auch in Löbau. Ein ungenügender Dienstleistungssektor verringert die Einnahmen aus dem Tourismus. Ein Teufelskreis, den die Region wahrscheinlich nicht alleine durchbrechen kann.

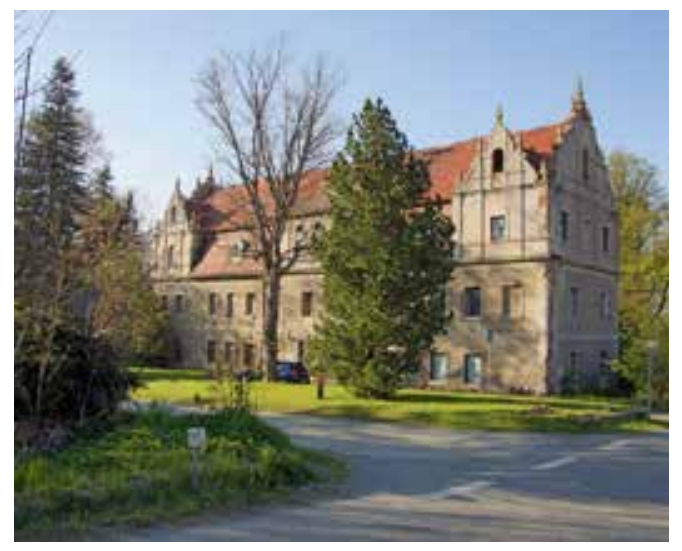

Die Bevölkerungsentwicklung der Stadt Löbau mit den eingemeindeten Ortsteilen seit 1834
Georgewitz (links), Schloss Bellwitz (rechts)

Fotos: Uwe Ulrich Jäschke, 2017

\section{Autor}

Prof. Dr. Uwe Ulrich Jäschke Hochschule für Technik und Wirtschaft Dresden Fakultät Geoinformation Friedrich-List-Platz 1 01069 Dresden 